\title{
Glutamatergic Metabolites, Volume and Cortical Thickness in Antipsychotic-Naive Patients with First-Episode Psychosis: Implications for Excitotoxicity
}

\author{
Eric Plitman ${ }^{1,2}$, Raihaan Patel ${ }^{3,4}$, Jun Ku Chung ${ }^{1,2}$, Jon Pipitone ${ }^{5}$, Sofia Chavez ${ }^{1,6}$, Francisco Reyes-Madrigal ${ }^{7}$, \\ Gladys Gómez-Cruz ${ }^{7}$, Pablo León-Ortiz ${ }^{7,8}$, M Mallar Chakravarty ${ }^{3,4}$, Camilo de la Fuente-Sandoval ${ }^{*, 7,9}$ and \\ Ariel Graff-Guerrero $1,2,6,10,11$
}

\begin{abstract}
'Multimodal Imaging Group, Research Imaging Centre, Centre for Addiction and Mental Health, University of Toronto, Toronto, ON, Canada;
${ }^{2}$ Institute of Medical Science, University of Toronto, Toronto, ON, Canada; ${ }^{3}$ Cerebral Imaging Centre, Douglas Mental Health University Institute, McGill University, Montreal, QC, Canada; ${ }^{4}$ Departments of Psychiatry and Biomedical Engineering, McGill University, Montreal, QC, Canada; ${ }^{5}$ Kimel Family Translational Imaging-Genetics Research Laboratory, Research Imaging Centre, Centre for Addiction and Mental Health, University of Toronto, Toronto, ON, Canada; ${ }^{6}$ Department of Psychiatry, University of Toronto, Toronto, ON, Canada; ' Laboratory of Experimental Psychiatry, Instituto Nacional de Neurología y Neurocirugía, Mexico City, Mexico; ${ }^{8}$ Department of Education, Instituto Nacional de Neurología y Neurocirugía, Mexico City, Mexico; ${ }^{9}$ Neuropsychiatry Department, Instituto Nacional de Neurología y Neurocirugía, Mexico City, Mexico; ${ }^{10}$ Geriatric Mental Health Division, Centre for Addiction and Mental Health, University of Toronto, Toronto, ON, Canada; "Campbell Institute Research Program, Centre for Addiction and Mental Health, University of Toronto, Toronto, ON, Canada
\end{abstract}

\begin{abstract}
Neuroimaging studies investigating patients with schizophrenia often report appreciable volumetric reductions and cortical thinning, yet the cause of these deficits is unknown. The association between subcortical and cortical structural alterations, and glutamatergic neurometabolites is of particular interest due to glutamate's capacity for neurotoxicity; elevated levels may be related to neuroanatomical compromise through an excitotoxic process. To this end, we explored the relationships between glutamatergic neurometabolites and structural measures in antipsychotic-naive patients experiencing their first non-affective episode of psychosis (FEP). Sixty antipsychoticnaive patients with FEP and 60 age- and sex-matched healthy controls underwent a magnetic resonance imaging session, which included a TI-weighted volumetric image and proton magnetic resonance spectroscopy in the precommissural dorsal caudate. Group differences in precommissural caudate volume (PCV) and cortical thickness (CT), and the relationships between glutamatergic neurometabolites (ie, glutamate+glutamine (Glx) and glutamate) and these structural measures, were examined. PCV was decreased in the FEP group $(p<0.00$ I), yet did not differ when controlling for total brain volume. Cortical thinning existed in the FEP group within frontal, parietal, temporal, occipital, and limbic regions at a 5\% false discovery rate. Glx levels were negatively associated with PCV only in the FEP group $(p=0.0$ 18). The observed relationship between Glx and PCV in the FEP group is supportive of a focal excitotoxic mechanism whereby increased levels of glutamatergic markers are related to local structural losses. This process may be related to the prominent structural deficits that exist in patients with schizophrenia.
\end{abstract}

Neuropsychopharmacology (2016) 4I, 2606-26I3; doi:I0.1038/npp.2016.84; published online 29 June 2016

\section{INTRODUCTION}

Previous studies have identified appreciable volumetric deficits and cortical thinning in patients with schizophrenia (Rimol et al, 2010; van Erp et al, 2016). However, chronicity and medication intake may confound the assessment of these measures and thus true illness pathophysiology. The investigation of antipsychotic-naive patients experiencing

*Correspondence: Dr C de la Fuente-Sandoval, Laboratory of Experimental Psychiatry and Neuropsychiatry Department, Instituto Nacional de Neurología y Neurocirugía, Insurgentes Sur 3877, La Fama, Tlalpan, I4269 Mexico City, Mexico, Tel: +52 5556063822 (ext. 5059), Fax: +52 55 5171 6456, E-mail: fcamilo@unam.mx

Received 30 January 20 16; revised 6 May 20 16; accepted 27 May 20 I6; accepted article preview online 8 June 2016 their first episode of psychosis (FEP) is beneficial in that confounding effects are substantially reduced. Previous meta-analyses have specifically identified volumetric deficits in patients with FEP (Ellison-Wright et al, 2008; Olabi et al, 2012) and in antipsychotic-naive patients with schizophrenia (Haijma et al, 2013). Similarly, cortical thickness (CT) alterations have been observed in antipsychotic-naive patients with FEP (Song et al, 2015; Xiao et al, 2015). However, the mechanisms through which these structural changes occur are presently unknown.

Glutamate is an abundant excitatory neurotransmitter that exists in large intracellular concentrations in the brain (Mehta et al, 2013). When present in abnormally high extracellular concentrations, glutamate may have a neurotoxic effect through a process referred to as excitotoxicity, 
whereby overstimulation by glutamate increases intracellular calcium and triggers a cascade of events that lead to cell death (Lahti and Reid, 2011; Mehta et al, 2013). Several studies have quantified glutamate in patients with schizophrenia using proton magnetic resonance spectroscopy $\left({ }^{1} \mathrm{H}-\mathrm{MRS}\right)$. Studies investigating patients in the early stages of the disorder, in which subjects are either antipsychoticnaive or have been minimally exposed to antipsychotic medication, have observed increased levels of glutamatergic neurometabolites (de la Fuente-Sandoval et al, 2013, 2011; Kegeles et al, 2012; Kraguljac et al, 2013; Plitman et al, 2016); this increase may have an excitotoxic effect in several brain regions. However, as previously reviewed by our group, only a few human studies currently exist that have investigated the relationship between glutamatergic neurometabolites and measures of brain structure, collectively providing inconclusive support for the role of glutamate-mediated excitotoxicity in the structural deficits present in schizophrenia (Plitman et al, 2014). Our group has also previously reported increases in glutamate and glutamate+glutamine (Glx) levels in patients with FEP within the precommissural dorsal caudate (PDC) (de la Fuente-Sandoval et al, 2013, 2011; Plitman et al, 2016), an area highly implicated in the pathophysiology of schizophrenia that has been reported to specifically contain communications with cortical brain regions (Kegeles et al, 2010).

Given the overwhelming evidence for structural compromise in patients with schizophrenia, there is a pressing need to better understand the role of glutamate-mediated excitotoxicity in neuroanatomical alteration. To answer this question, this study examined the relationships between levels of glutamatergic neurometabolites (ie, glutamate and $\mathrm{Glx}$ ) in the PDC and structural measures (ie, precommissural caudate volume (PCV) and CT) in the largest sample to date of antipsychotic-naive patients with FEP in which ${ }^{1} \mathrm{H}$-MRS was performed. The study of PCV provides a specific and local examination of this phenomenon, as the ${ }^{1} \mathrm{H}$-MRS voxel was preferentially located within the PDC, while CT assesses a more global excitotoxic mechanism. To the best of our knowledge, no previous study has investigated these relationships. On the basis of previous literature (Ellison-Wright et al, 2008; Haijma et al, 2013; Olabi et al, 2012; Song et al, 2015; Xiao et al, 2015), we hypothesized that the FEP group would demonstrate PCV deficits in addition to widespread cortical thinning. Also, in accordance with a local excitotoxic process (Kraguljac et al, 2013; Plitman et al, 2014), we hypothesized that increased levels of glutamatergic neurometabolites would be associated with PCV loss in the patient group, while no such relationship would exist with CT. Finally, as an exploratory investigation, we examined the relationships between structural measures and clinical symptoms, hypothesizing that lower PCV and cortical thinning would be related to higher symptom scores.

\section{MATERIALS AND METHODS}

\section{Participants, Clinical Assessment, Magnetic Resonance Studies, and ${ }^{1}$ H-MRS Data Analysis}

Information concerning participants, ethical approval, clinical assessment, imaging parameters, and ${ }^{1} \mathrm{H}$-MRS data analysis has been presented within a previous manuscript (Plitman et al, 2016); however, detailed methodology is included within the Supplementary Information. The present study included 60 antipsychotic-naive patients with FEP and 60 age- and sex-matched healthy controls. ${ }^{1} \mathrm{H}-\mathrm{MRS}$ spectra were acquired in the right PDC using an 8-ml isotropic voxel, point-resolved spectroscopy, and a TE of $35 \mathrm{~ms}$. Water suppressed spectra were analyzed with LCModel version 6.3-0E (Provencher, 2001). Spectra were normalized to the unsuppressed water signal. Spectroscopic values were corrected for cerebrospinal fluid (CSF) fraction within the ${ }^{1} \mathrm{H}-\mathrm{MRS}$ voxel (de la Fuente-Sandoval et al, 2011). A \%SD of $20 \%$ or greater, or a full-width at half maximum (FWHM) value exceeding $12 \mathrm{~Hz}$, resulted in exclusion. Psychopathology was assessed using the Positive and Negative Syndrome Scale (PANSS) (Kay et al, 1987).

\section{Image Preprocessing}

All structural imaging analysis was done in the minc format. T1-weighted structural images were first preprocessed using an intensity correction tool. Intensity correction was performed using the N4ITK algorithm (Tustison et al, 2010), an improved version of the popular N3 correction algorithm (Sled et al, 1998). This updated version provides improved bias field correction via a robust B-spline approximation routine with the capability to handle a range of resolutions. It also provides an optimization scheme that leads to better convergence and a more accurate estimation of the overall bias field (Tustison et al, 2010).

\section{PCV Analysis}

For volume analyses, T1-weighted structural images were additionally preprocessed using an autocrop tool part of the minc toolkit (https://github.com/BIC-MNI/mni_autoreg), which resampled each image to a $1-\mathrm{mm}$ isotropic slicing. This resampling was done in order to satisfy computational resource limits, as well as to achieve consistency throughout the data set. Note that this is a stage that is carried out in various other image processing tool chains (Lerch and Evans, 2005). Fully-automated segmentation of striatal subdivisions was carried out using the Multiple Automatically Generated Templates (MAGeT-Brain) algorithm (Chakravarty et al, 2013). This technique is a modified multi-atlas segmentation technique designed to use a limited number of high-quality manually segmented atlases as an input. In this case, the striatal subdivisions atlas-based on a histology-based atlas warped to fit the Colin-27 Subcortical Atlas (Chakravarty et al, 2006)—-was used as the single atlas input. A subset of the population under study is used as a template library through which the final segmentation is bootstrapped. Each subject in the template library is segmented through nonlinear atlas-to-template registration followed by label propagation, yielding a unique definition of the subdivisions for each of the templates. For this work, 21 templates were used from the overall subject pool. A matched set of 10 patients with FEP and 11 healthy controls were chosen so as to ensure a representative template set. The bootstrapping of the final segmentations through the template library results in 21 candidate labels produced for each subject and labels are then fused using a majority vote to complete the segmentation process. Non-linear registration was performed using a version of the Automatic Normalization Tools (ANTS) 
registration technique (Avants et al, 2008) that is compatible with the minc toolkit (https://github.com/vfonov/mincANTS). To focus on most local phenomena, only right PCV was examined in primary analyses, given that the ${ }^{1} \mathrm{H}$-MRS voxel was preferentially placed in the right PDC (Supplementary Figure S1).

\section{Total Brain Volume Analysis}

Total brain volume (TBV) was obtained using the Brain Extraction based on non-local Segmentation Technique (BEaST) method (Eskildsen et al, 2012), which is based on non-local segmentation in a multi-resolution framework. In BEaST, each voxel is labeled based on the similarity of its neighborhood of voxels to all the neighborhoods in a library of pre-defined priors and a non-local means estimator is used to estimate the label at the voxel. Inputs are downsampled to a lower resolution, segmentation is performed, and results are propagated up to higher resolutions (Eskildsen et al, 2012). BEaST is designed to include CSF (in the ventricles, cerebellar cistern, deep sulci, along surface of brain, and brainstem), the brainstem, and cerebellar white matter (WM) and gray matter (GM) in the brain mask, while excluding the skull, skin, fat, muscles, dura, eyes, bone, exterior blood vessels, and exterior nerves. Images preprocessed for MAGeT were similarly used for BEaST.

\section{CT Analysis}

CT was estimated using the CIVET processing pipeline (version 1.1.12; Montreal Neurological Institute). T1-weighted images were aligned linearly to the ICBM 152 average template through a nine-parameter transformation (three translations, rotations, and scales) (Collins et $a l, 1994)$ and preprocessed to reduce intensity non-uniformity effects (Sled et al, 1998). Next, images were classified into GM, WM, and CSF (Zijdenbos et al, 2002). Hemispheres were then modeled as GM and WM surfaces using a deformable model strategy, which generates 4 separate surfaces, each defined by 40962 vertices (Kim et al, 2005). CT was determined in native space through non-linear surfacebased normalization that uses a midsurface between pial and WM surfaces. Images were then smoothed with a $20-\mathrm{mm}$ surface-based diffusion kernel and non-linearly registered to a minimally biased surface-based template (Boucher et al, 2009). Native-space thicknesses were used in all analyses, considering that normalizing for head or brain volume has little relationship to $\mathrm{CT}$ and risks introducing noise.

\section{Statistical Analysis}

Analyses were performed using SPSS Statistics version 21 (IBM Corporation). Independent-sample $t$ tests were used to compare demographic characteristics, Cramer-Rao lower bounds (CRLBs), FWHM values, signal-to-noise ratios, and GM, WM, and CSF percentages between groups. $\chi^{2}$ or Fisher's exact tests were used to compare frequency data. Glutamatergic neurometabolite levels were compared between groups using analyses of variance.

Group differences in TBV were assessed using an analysis of covariance (ANCOVA), controlling for original T1-weighted image voxel dimensions (henceforth, dimensions), age, and sex. Group differences in PCV were assessed with an ANCOVA, controlling for dimensions, TBV, age, and sex; here, analyses were also performed without TBV as a covariate. Additionally, multiple regressions were performed separately for each group to investigate the relationships between glutamatergic neurometabolite levels and PCV, controlling for dimensions, TBV, age, and sex. Finally, multiple regressions were utilized to measure the relationships between PCV and PANSS subscale total scores, controlling for dimensions, TBV, age, and sex. Owing to our a priori hypotheses that PCV would be smaller in the FEP group and that glutamatergic neurometabolites would have a negative relationship with PCV in the FEP group, significance thresholds set at $p<0.05$ and $p<0.025(p=0.05 \div 2$ to correct for the examination of both glutamate and $\mathrm{Glx}$ ), respectively, were used for these investigations. The assessment of the relationships between PCV and clinical symptoms was considered exploratory; thus, a Bonferroni correction for multiple comparisons was employed.

CT vertexwise analyses were performed with the RMINC package (https://github.com/mcvaneede/RMINC). Using a general linear model, separate CT regression analyses were carried out for diagnosis, glutamate, Glx, and PANSS subscale total scores, each controlling for dimensions, age, and sex. Glutamate and Glx analyses were performed independently for FEP and control groups. Maps of $t$ statistics at each vertex were projected onto an average brain template. Analyses were corrected for multiple comparisons using a false discovery rate (FDR $<0.05)$.

Outliers were defined as greater than three times the interquartile range; where applicable, outliers were removed in a neurometabolite-specific manner.

\section{RESULTS}

\section{Demographic, Clinical, and ${ }^{1} \mathrm{H}-\mathrm{MRS}$ Group Differences}

Demographic, clinical, and ${ }^{1} \mathrm{H}-\mathrm{MRS}$ results have been reported upon in another publication (Plitman et al, 2016), although data pertinent to the present study are included in Supplementary Tables S1-S3. DSM-IV diagnoses of patients with FEP were brief psychotic disorder $(n=14)$, schizophreniform disorder $(n=21)$, and schizophrenia $(n=25)$. No group differences existed in age, sex, handedness, and cannabis use. Education years were higher in the control group $(t(118)=6.40, p<0.001)$, whereas tobacco use was greater in the FEP group $\left(\chi^{2}=5.21, p=0.039\right)$. The FEP group had a mean duration of untreated psychosis of $33.03 \pm 52.70$ weeks, and mean PANSS positive, negative, and general psychopathology subscale total scores of $24.13 \pm 4.97,24.33 \pm 5.66$, and $48.75 \pm 8.38$, respectively. One glutamate outlier was removed before analysis; however, removal of this outlier did not affect results. Glutamate levels were higher in the FEP group $(\mathrm{F}(1,117)=11.63, p<0.001)$, whereas Glx levels were not different between groups $(\mathrm{F}(1,118)=1.84, p=0.18)$. Neurometabolite CRLBs, FWHM values, signal-to-noise ratios, and GM, WM, and CSF voxel percentages did not differ between groups.

\section{Group Differences in PCV and TBV}

No outliers were identified for PCV or TBV. PCV and TBV values are presented in Table 1 ; mean PCV values are also 
Table I Precommissural Caudate Volume and Total Brain Volume in Patients with First-Episode Psychosis and Healthy Controls

\begin{tabular}{lcc}
\hline & \multicolumn{2}{c}{ Mean (SD) $\left(\mathbf{m m}^{\mathbf{3}}\right)$} \\
\cline { 2 - 3 } & PCV & TBV \\
\hline FEP group & $2109.80(320.91)^{\mathrm{a}}$ & $\mid 354333.20(142317.05)^{\mathrm{a}}$ \\
HC group & $2302.63(287.98)$ & $\mid 442801.00(137349.56)$ \\
\hline
\end{tabular}

Abbreviations: FEP, first-episode psychosis; HC, healthy control; PCV,

precommissural caudate volume; TBV, total brain volume.

${ }^{\mathrm{a} D e n o t e s} p<0.001$.

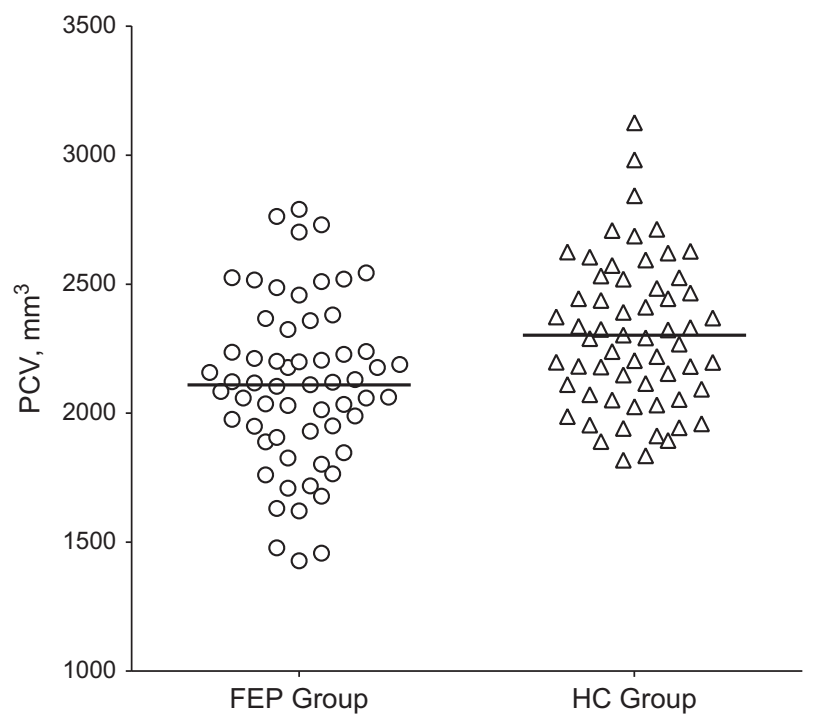

Figure I Precommissural caudate volume (PCV) in patients with firstepisode psychosis and healthy controls.

displayed in Figure 1. Without TBV as a covariate, PCV was smaller in the FEP group $(\mathrm{F}(1,114)=11.88, p<0.001)$. However, controlling for TBV rendered the group difference insignificant $(\mathrm{F}(1,113)=1.18, p=0.28)$. TBV was smaller in the FEP group $(\mathrm{F}(1,114)=16.80, p<0.001)$.

\section{Group Differences in CT}

Widespread thinning was identified in the FEP group within the frontal gyri (right superior, bilateral middle, bilateral inferior, bilateral precentral), parietal gyri (bilateral postcentral, bilateral supramarginal, bilateral angular), temporal gyri (bilateral superior, right middle, right inferior), bilateral gyrus rectus, bilateral orbitofrontal cortex, bilateral precuneus, bilateral cuneus, right superior parietal lobule, right paracentral lobule, right parahippocampal gyrus, right lingual gyrus, and right fusiform gyrus (Figure 2). CT was not increased at any vertex in the FEP group.

\section{Relationships Between Neurometabolite Levels and Structural Measures}

The relationships between glutamatergic neurometabolite levels and PCV are presented in Table 2. Glutamate levels were not related to PCV in either group. Glx levels were negatively associated with PCV only in the FEP group $(\beta=-0.25, t=-2.44, p=0.018)$. The relationship between $\mathrm{PCV} / \mathrm{TBV}$ and Glx levels in the FEP group is displayed in Figure 3. No relationships were identified between glutamatergic markers and CT in either group.

\section{Relationships Between Clinical Symptoms and Structural Measures}

No relationships between PANSS subscale total scores and PCV were identified (Supplementary Table S5). Similarly, no relationships between PANSS subscale total scores and CT were identified.

\section{DISCUSSION}

The present study aimed to investigate whether glutamatergic markers in the PDC were related to PCV and CT in a sample of antipsychotic-naive patients with FEP. Expectedly, we found decreased PCV and widespread cortical thinning within the FEP group; however, when TBV was included as a covariate, PCV did not differ between groups. Glx levels were negatively associated with PCV in the FEP group, while no relationships involving glutamate or CT were identified.

To the best of our knowledge, this is the first study to examine the relationships between glutamatergic neurometabolite levels and volume in this population within the precommissural caudate. Also, though MAGeT-Brain has been recently used to investigate patients with childhoodonset schizophrenia and their non-psychotic siblings (Chakravarty et al, 2015), the present study is the first to use this algorithm to assess a measure of striatal volume in an antipsychotic-naive sample of patients with FEP. Likewise, we believe this is the first study to investigate the relationships between glutamatergic neurometabolite levels and CT in patients with FEP or schizophrenia.

Our finding of decreased PCV in the FEP group is consistent with the current literature. Two meta-analyses found bilateral caudate head GM volume decreases in patients with FEP (Ellison-Wright et al, 2008; Olabi et al, 2012), one of which identified that deficits were more pronounced in FEP than in chronic schizophrenia (Ellison-Wright et al, 2008). Another meta-analysis reported that caudate nucleus reductions were greater in antipsychotic-naive patients than in medicated patients with schizophrenia (Haijma et al, 2013). Caudate volume reductions have been similarly observed in studies including antipsychotic-naive patients with FEP that have adjusted for TBV-analogous measures (Ebdrup et al, 2010; Keshavan et al, 1998). However, other studies considering the effect of TBV have shown no caudate volumetric deficits in antipsychotic-naive or minimally treated patients with FEP (Glenthoj et al, 2007; Gunduz et al, 2002). In our study, group differences in PCV failed to reach significance once TBV was considered as a covariate, suggesting that group volumetric differences may not be specific to PCV and might be occurring simultaneously in other brain regions, such that controlling for TBV masks the PCV deficit in the patient population.

Only one prior study has investigated striatal subdivision volumes in patients with schizophrenia; this study examined medicated male patients with chronic schizophrenia and 

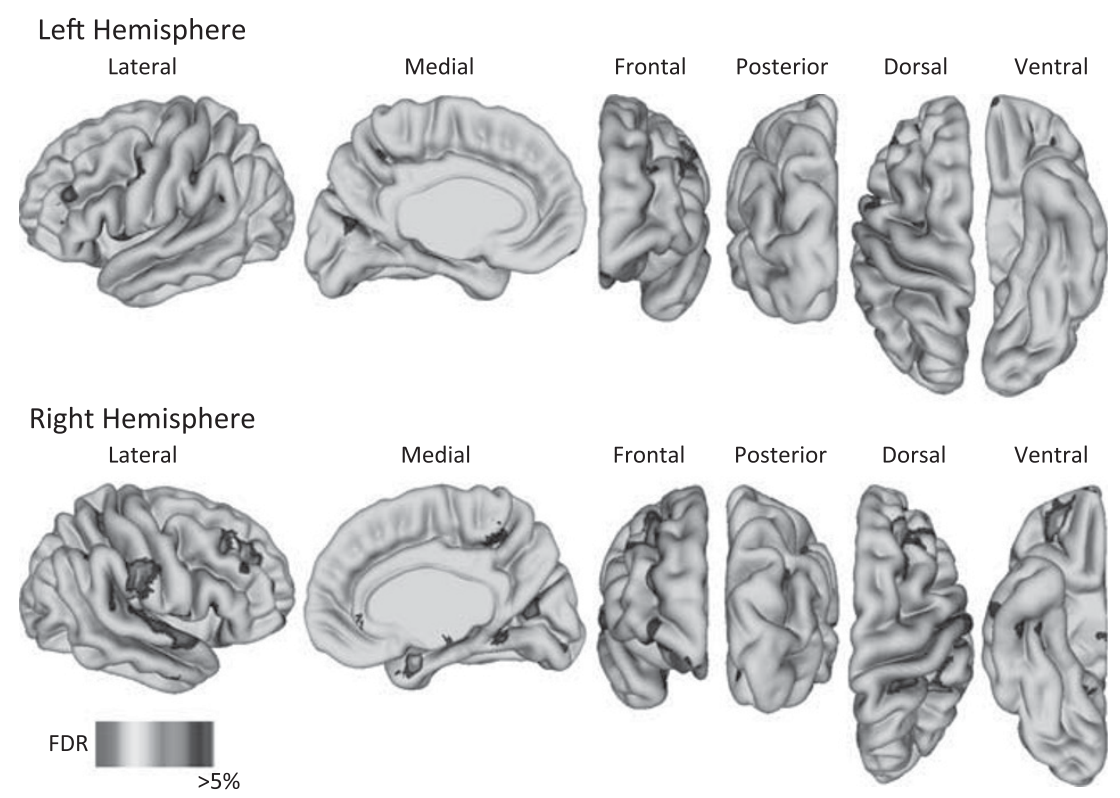

Figure 2 Cortical thinning in patients with first-episode psychosis compared with healthy controls. Abbreviation: FDR, false discovery rate.

Table 2 Relationships Between Glutamatergic Neurometabolite Levels and Precommissural Caudate Volume ${ }^{a}$

\begin{tabular}{lcc}
\hline Variable & Glu & Glx \\
\hline FEP group & \\
$P C V$ & $\beta=-0.08, t=-0.81, p=0.42$ & $\beta=-0.25, t=-2.44, p=0.018^{\mathrm{b}, \mathrm{c}}$ \\
HC group & & \\
$P C V$ & $\beta=0.1 \mathrm{I}, t=0.88, p=0.38$ & $\beta=0.01, t=0.09, p=0.93$ \\
\hline
\end{tabular}

Abbreviations: FEP, first-episode psychosis; Glu, glutamate; Glx, glutamate + glutamine; HC, healthy control; PCV, precommissural caudate volume.

a For ease of presentation, only model statistics for significant relationships are included; others are in Supplementary Table S4.

bModel statistics: adjusted $R^{2}=0.597, F_{5.54}=|8.5|, p<0.00 \mid$.

${ }^{\mathrm{c}}$ Denotes $p<0.05$.

found no reduction in PDC volume relative to total intracranial contents (Levitt et al, 2013). This is in line with a recent meta-analysis that found no caudate volumetric abnormalities in a large sample of mostly medicated patients with schizophrenia regardless whether intracranial volume was included as a covariate (van Erp et al, 2016). Of note, in this meta-analysis, the sample proportions of medicationnaive patients were not associated with caudate volume.

Furthermore, the PDC has been reported to have elevated dopaminergic function and is highly implicated in the pathophysiology of schizophrenia (Kegeles et al, 2010). Excitotoxicity in this region may impact dopaminergic activity and thereby contribute to symptomatology. In the present study, we observed a negative relationship between Glx levels in the PDC and PCV in the FEP group, providing support for a local excitotoxic process. Glutamate-mediated excitotoxicity may contribute to the neuroanatomical deviations present in schizophrenia. It is currently posited that hypofunctioning $\mathrm{N}$-methyl-D-aspartate receptors (NMDARs) on gammaaminobutyric acid-ergic inhibitory interneurons lead to the disinhibition of downstream pyramidal neurons, a

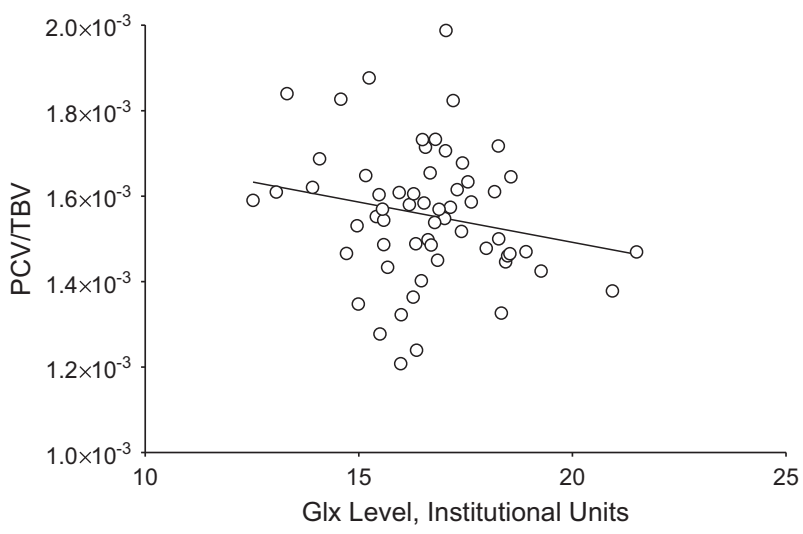

Figure 3 Relationship between glutamate+glutamine (Glx) levels and precommissural caudate volume (PCV) relative to total brain volume (TBV) in patients with first-episode psychosis.

subsequent increase in glutamate release, and consequent excitotoxicity (Stone et al, 2007). Evidence for this phenomenon arises from pharmacological studies using NMDAR antagonists, which lead to the emergence of schizophrenialike symptomatology in healthy volunteers (Krystal et al, 1994; Lahti et al, 2001) and elicit symptom exacerbation in patients with schizophrenia (Lahti et al, 1995, 2001). Studies in which rodents are exposed to an NMDAR antagonist have shown elevations in extracellular glutamatergic markers (Adams and Moghaddam, 1998; Bustos et al, 1992). Comparably, ${ }^{1} \mathrm{H}$-MRS studies in healthy humans have shown increased glutamate and glutamine following acute exposure to ketamine (Rowland et al, 2005; Stone et al, 2012). Moreover, studies administering an NMDAR antagonist to rodents have demonstrated resultant neurotoxic injury, which resembles features of schizophrenia and is attenuated by agents inhibiting glutamate release (Plitman et al, 2014).

While preclinical literature strongly supports the role of glutamate-mediated excitotoxicity in schizophrenia, human 
studies have provided insufficient evidence for the phenomenon (Plitman et al, 2014). Kraguljac et al (2013) reported a negative relationship between left hippocampal Glx and two clusters of left hippocampal GM volume adjusted for total intracranial volume, only in the unmedicated patient group and not in controls. However, Klar et al (2010) did not find a relationship between hippocampal glutamate and hippocampal volume in a medicated sample. Two longitudinal studies investigating a sample of initially unmedicated patients who were then medicated at follow-up visits noted positive correlations between decreasing thalamic glutamate and GM volume within frontal, temporal, parietal, and limbic areas (Aoyama et al, 2011; Theberge et al, 2007). The observed negative relationship between Glx levels and local PCV in the present study is most comparable to the findings of Kraguljac et al (2013) and supports an excitotoxic process in schizophrenia. Our analysis similarly included TBV as a covariate, suggesting that whole brain effects do not drive the relationship between Glx and PCV, and indicating a localized effect. However, the lack of a group difference in PCV when TBV was included as a covariate is not wholly supportive of a specific excitotoxic process in this brain region.

Notably, Glx levels were not significantly elevated in the FEP group, though mean levels were higher than the control group; this group difference may have been underpowered to achieve statistical significance. Thus, the proposed excitotoxicity in the patient group might be a result of elevated Glx levels or a pathological process specific to individuals with FEP that increases susceptibility to the excitotoxicity phenomenon. It is unclear why a similar relationship did not exist for glutamate levels, which were significantly higher in the FEP group. We speculate that glutamine's influence on the Glx peak had a contributing effect; abnormalities in glutamatergic metabolism might account for the observed results in addition to or instead of excitotoxicity, especially considering that ${ }^{1} \mathrm{H}-\mathrm{MRS}$ cannot distinguish synaptic from extra-synaptic pools of neurometabolites. Further, neurotransmitter glutamate likely has a greater role in excitotoxicity than intracellular glutamate, and it has previously been suggested that glutamine (contained in Glx) may more robustly reflect the neurotransmitter pool of glutamate than ${ }^{1} \mathrm{H}$-MRS glutamate itself, which may be mainly intracellular (Wijtenburg et al, 2015).

The cortical thinning identified in the FEP group was diffuse, involving frontal, parietal, temporal, occipital, and limbic brain regions. Our findings are akin to those of previous studies investigating CT in antipsychotic-naive patients with FEP, in which widespread thinning has been reported (Song et al, 2015; Xiao et al, 2015). Within our investigation, the lack of any observed relationships between glutamatergic markers and CT suggests that PDC glutamatergic activity does not account for widespread cortical thinning. Thus, cortical thinning might occur through an alternate mechanism. However, glutamatergic neurometabolites were not measured in cortical areas; consequently, a local inverse relationship between cortical glutamatergic neurometabolites and cortical structural measures cannot be excluded.

Finally, neither PCV nor CT was related to any PANSS subscale total score. Previous studies have similarly failed to identify an association between caudate volumes and symptom scores in untreated patients with schizophrenia
(Ballmaier et al, 2008; Venkatasubramanian et al, 2003). With respect to $\mathrm{CT}$, while this null finding is comparable to those from previous CT investigations of patients with FEP or chronic schizophrenia (Rimol et al, 2010; Song et al, 2015), it contrasts a recent CIVET examination of antipsychotic-naive FEP patients in which cortical thinning within frontal regions was related to higher PANSS-positive symptom scores (Xiao et al, 2015).

This study must be considered in light of its limitations. First, the adopted study design does not provide insight toward illness progression or causation. Second, tobacco use was greater in the FEP group. Importantly, when tobacco use was examined as a covariate in all analyses, the only deviation from the presented results was additional cortical thinning observed in the patient group within the left parahippocampal gyrus. Third, to reduce imaging time in patients with active psychosis, ${ }^{1} \mathrm{H}$-MRS was only performed in the right $\mathrm{PDC}$, although glutamatergic neurometabolite laterality differences were not previously observed in this region (de la Fuente-Sandoval et al, 2009). Fourth, the inclusion of patients with brief psychotic disorder presents an important limitation given their short duration of illness. Fifth, since the ${ }^{1} \mathrm{H}-\mathrm{MRS}$ voxel was located in the right PDC, the present study focused on the examination of right PCV. However, an exploratory analysis involving left PCV demonstrated no laterality effects: left PCV was reduced in the FEP group without TBV as a covariate $(\mathrm{F}(1,114)=8.27$, $p=0.005)$; controlling for TBV rendered this difference insignificant; Glx levels were negatively associated with left PCV only in the FEP group $(\beta=-0.23, t=-2.17, p=0.035$; Model Statistics: adjusted $\left.R^{2}=0.541, \mathrm{~F}_{5,54}=14.91, p<0.001\right)$; and no relationships existed between left PCV and glutamate or PANSS subscale total scores. Sixth, despite the preferential placement of the ${ }^{1} \mathrm{H}-\mathrm{MRS}$ voxel in the PDC, it encompassed other associative striatum subregions and included components of other structures (eg, putamen and internal capsule). The ${ }^{1} \mathrm{H}$-MRS voxel location was referred to as the PDC to maintain consistency in nomenclature. Seventh, to focus on most local phenomena and examine a highly implicated subregion in schizophrenia, the current investigation employed an a priori hypothesis concerning the precommissural caudate. Although the present study is limited in its investigation of regionally specific excitotoxicity in that ${ }^{1} \mathrm{H}$-MRS was not collected elsewhere, other subcortical structure volumes were examined in exploratory analyses to provide support for this phenomenon. As shown in Supplementary Tables S6 and S7, no associations were identified between PDC glutamatergic neurometabolites and other subcortical structure volumes after correcting for multiple comparisons. Eighth, with PRESS at 3T, chemical shift artifact might result in varying volumes of interest between neurometabolites and the water signal; however, we anticipate that such would be the case for both patients and controls. Finally, though our sample size was not small, our study may have been underpowered.

Taken together, our findings offer evidence in support of a local excitotoxic process in schizophrenia. This mechanism may contribute to structural compromise in the illness. Going forward, a longitudinal study following FEP patients as they transition to schizophrenia, investigating several brain regions using ${ }^{1} \mathrm{H}-\mathrm{MRS}$, is warranted. An improved understanding of dysregulated neurometabolites and their 
consequent impact on structural losses in schizophrenia may aid in furthering our knowledge of illness pathophysiology and might identify future diagnostic and therapeutic strategies.

\section{FUNDING AND DISCLOSURE}

Computations were performed on the gpc supercomputer at the SciNet HPC Consortium. SciNet is funded by the Canada Foundation for Innovation under the auspices of Compute Canada, the Government of Ontario, Ontario Research Fund-Research Excellence, and the University of Toronto.

\section{ACKNOWLEDGMENTS}

We would like to acknowledge the technical assistance provided by Gabriel Devenyi and Robert Amaral, in addition to the invaluable support provided by the entire CoBrA Lab at the Douglas Mental Health University Institute, Montreal, QC, Canada. We would also like to acknowledge the important and frequent assistance provided by Shinichiro Nakajima, Yusuke Iwata, Fernando Caravaggio, and Philip Gerretsen. This work was supported by Consejo Nacional de Ciencia y Tecnología (CONACyT) (182279 to CdlF-S and AG-G), CONACyT project 261895 (CdlF-S), the CONACyT Scholarship (FR-M and PL-O), the Sistema Nacional de Investigadores (CdlF-S and AG-G), the Canadian Institute of Health Research (CIHR) (MOP-114989 to AG-G), the Vanier Canada Graduate Scholarship (EP), the Ontario Graduate Scholarship (EP), and the Canada Graduate Scholarship - Master's (EP). These sources were not involved in the study design, data collection, data analysis, or the decision to publish the manuscript. EP has received funding from the Vanier Canada Graduate Scholarship, the Ontario Graduate Scholarship, and the Canada Graduate Scholarship - Master's. FR-M has served as a speaker for AstraZeneca. CdlF-S has received support from the United States National Institute of Health (US-NIH), CONACyT, the Instituto de Ciencia y Tecnología del DF (ICyTDF), Janssen, AstraZeneca, and Eli Lilly. AG-G has received support from US-NIH, CIHR, the Ontario Mental Health Foundation, CONACyT, ICyTDF, the Brain \& Behavior Research Foundation (Formerly NARSAD), the Ontario Ministry of Health and Long-Term Care, the Ontario Ministry of Research and Innovation Early Research Award, and Janssen. EP, MMC, CdlF-S, and AG-G contributed to the study concept and design. FR-M, GG-C, PL-O, CdlF-S, and AG-G participated in data acquisition. EP, RP, JKC, JP, SC, MMC, CdlF-S, and AG-G analyzed and interpreted the data. EP, RP, MMC, CdlF-S, and AG-G drafted the manuscript. All authors critically revised the manuscript. SC provided technical support. CdlF-S and AG-G supervised the study. All authors have approved the final version of this manuscript and have agreed to its submission.

\section{REFERENCES}

Adams B, Moghaddam B (1998). Corticolimbic dopamine neurotransmission is temporally dissociated from the cognitive and locomotor effects of phencyclidine. J Neurosci 18: 5545-5554.
Aoyama N, Theberge J, Drost DJ, Manchanda R, Northcott S, Neufeld RW et al (2011). Grey matter and social functioning correlates of glutamatergic metabolite loss in schizophrenia. Br J Psychiatry 198: 448-456.

Avants BB, Epstein CL, Grossman M, Gee JC (2008). Symmetric diffeomorphic image registration with cross-correlation: evaluating automated labeling of elderly and neurodegenerative brain. Med Image Anal 12: 26-41.

Ballmaier M, Schlagenhauf F, Toga AW, Gallinat J, Koslowski M, Zoli $\mathrm{M}$ et al (2008). Regional patterns and clinical correlates of basal ganglia morphology in non-medicated schizophrenia. Schizophr Res 106: 140-147.

Boucher M, Whitesides S, Evans A (2009). Depth potential function for folding pattern representation, registration and analysis. Med Image Anal 13: 203-214.

Bustos G, Abarca J, Forray MI, Gysling K, Bradberry CW, Roth RH (1992). Regulation of excitatory amino acid release by N-methyl$\mathrm{D}$-aspartate receptors in rat striatum: in vivo microdialysis studies. Brain Res 585: 105-115.

Chakravarty MM, Bertrand G, Hodge CP, Sadikot AF, Collins DL (2006). The creation of a brain atlas for image guided neurosurgery using serial histological data. NeuroImage 30: 359-376.

Chakravarty MM, Rapoport JL, Giedd JN, Raznahan A, Shaw P, Collins DL et al (2015). Striatal shape abnormalities as novel neurodevelopmental endophenotypes in schizophrenia: a longitudinal study. Hum Brain Mapp 36: 1458-1469.

Chakravarty MM, Steadman P, van Eede MC, Calcott RD, Gu V, Shaw $P$ et al (2013). Performing label-fusion-based segmentation using multiple automatically generated templates. Hum Brain Mapp 34: 2635-2654.

Collins DL, Neelin P, Peters TM, Evans AC (1994). Automatic 3D intersubject registration of MR volumetric data in standardized Talairach space. J Comput Assist Tomogr 18: 192-205.

de la Fuente-Sandoval C, Favila R, Alvarado P, Leon-Ortiz P, Diaz-Galvis L, Amezcua C et al (2009). [Glutamate increase in the associative striatum in schizophrenia: a longitudinal magnetic resonance spectroscopy preliminary study]. Gac Med Mex 145: 109-113.

de la Fuente-Sandoval C, Leon-Ortiz P, Azcarraga M, Stephano S, Favila R, Diaz-Galvis L et al (2013). Glutamate levels in the associative striatum before and after 4 weeks of antipsychotic treatment in first-episode psychosis: a longitudinal proton magnetic resonance spectroscopy study. JAMA Psychiatry 70: 1057-1066.

de la Fuente-Sandoval C, Leon-Ortiz P, Favila R, Stephano S, Mamo D, Ramirez-Bermudez J et al (2011). Higher levels of glutamate in the associative-striatum of subjects with prodromal symptoms of schizophrenia and patients with first-episode psychosis. Neuropsychopharmacology 36: 1781-1791.

Ebdrup BH, Glenthoj B, Rasmussen H, Aggernaes B, Langkilde AR, Paulson $\mathrm{OB}$ et al (2010). Hippocampal and caudate volume reductions in antipsychotic-naive first-episode schizophrenia. J Psychiatry Neurosci 35: 95-104.

Ellison-Wright I, Glahn DC, Laird AR, Thelen SM, Bullmore E (2008). The anatomy of first-episode and chronic schizophrenia: an anatomical likelihood estimation meta-analysis. $\mathrm{Am} \mathrm{J}$ Psychiatry 165: 1015-1023.

Eskildsen SF, Coupe P, Fonov V, Manjon JV, Leung KK, Guizard N et al (2012). BEaST: brain extraction based on nonlocal segmentation technique. NeuroImage 59: 2362-2373.

Glenthoj A, Glenthoj BY, Mackeprang T, Pagsberg AK, Hemmingsen RP, Jernigan TL et al (2007). Basal ganglia volumes in drug-naive first-episode schizophrenia patients before and after short-term treatment with either a typical or an atypical antipsychotic drug. Psychiatry Res 154: 199-208.

Gunduz H, Wu H, Ashtari M, Bogerts B, Crandall D, Robinson DG et al (2002). Basal ganglia volumes in first-episode schizophrenia and healthy comparison subjects. Biol Psychiatry 51: 801-808. 
Haijma SV, Van Haren N, Cahn W, Koolschijn PC, Hulshoff Pol HE, Kahn RS (2013). Brain volumes in schizophrenia: a metaanalysis in over 18000 subjects. Schizophr Bull 39: 1129-1138.

Kay SR, Fiszbein A, Opler LA (1987). The positive and negative syndrome scale (PANSS) for schizophrenia. Schizophr Bull 13: 261-276.

Kegeles LS, Abi-Dargham A, Frankle WG, Gil R, Cooper TB, Slifstein $\mathrm{M}$ et al (2010). Increased synaptic dopamine function in associative regions of the striatum in schizophrenia. Arch Gen Psychiatry 67: 231-239.

Kegeles LS, Mao X, Stanford AD, Girgis R, Ojeil N, Xu X et al (2012). Elevated prefrontal cortex gamma-aminobutyric acid and glutamateglutamine levels in schizophrenia measured in vivo with proton magnetic resonance spectroscopy. Arch Gen Psychiatry 69: 449-459.

Keshavan MS, Rosenberg D, Sweeney JA, Pettegrew JW (1998). Decreased caudate volume in neuroleptic-naive psychotic patients. Am J Psychiatry 155: 774-778.

Kim JS, Singh V, Lee JK, Lerch J, Ad-Dab'bagh Y, MacDonald D et al (2005). Automated 3-D extraction and evaluation of the inner and outer cortical surfaces using a Laplacian map and partial volume effect classification. NeuroImage 27: 210-221.

Klar AA, Ballmaier M, Leopold K, Hake I, Schaefer M, Bruhl R et al (2010). Interaction of hippocampal volume and $\mathrm{N}$-acetylaspartate concentration deficits in schizophrenia: a combined MRI and 1H-MRS study. NeuroImage 53: 51-57.

Kraguljac NV, White DM, Reid MA, Lahti AC (2013). Increased hippocampal glutamate and volumetric deficits in unmedicated patients with schizophrenia. JAMA Psychiatry 70: 1294-1302.

Krystal JH, Karper LP, Seibyl JP, Freeman GK, Delaney R, Bremner JD et al (1994). Subanesthetic effects of the noncompetitive NMDA antagonist, ketamine, in humans. Psychotomimetic, perceptual, cognitive, and neuroendocrine responses. Arch Gen Psychiatry 51: 199-214.

Lahti AC, Koffel B, LaPorte D, Tamminga CA (1995). Subanesthetic doses of ketamine stimulate psychosis in schizophrenia. Neuropsychopharmacology 13: 9-19.

Lahti AC, Reid MA (2011). Is there evidence for neurotoxicity in the prodromal and early stages of schizophrenia? Neuropsychopharmacology 36: 1779-1780.

Lahti AC, Weiler MA, Tamara Michaelidis BA, Parwani A, Tamminga CA (2001). Effects of ketamine in normal and schizophrenic volunteers. Neuropsychopharmacology 25: 455-467.

Lerch JP, Evans AC (2005). Cortical thickness analysis examined through power analysis and a population simulation. NeuroImage 24: 163-173.

Levitt JJ, Rosow LK, Nestor PG, Pelavin PE, Swisher TM, McCarley RW et al (2013). A volumetric MRI study of limbic, associative and sensorimotor striatal subregions in schizophrenia. Schizophr Res 145: 11-19.

Mehta A, Prabhakar M, Kumar P, Deshmukh R, Sharma PL (2013). Excitotoxicity: bridge to various triggers in neurodegenerative disorders. Eur J Pharmacol 698: 6-18.

Olabi B, Ellison-Wright I, Bullmore E, Lawrie SM (2012). Structural brain changes in first episode schizophrenia compared with fronto-temporal lobar degeneration: a meta-analysis. BMC Psychiatry 12: 104.

Plitman E, de la Fuente-Sandoval C, Reyes-Madrigal F, Chavez S, Gomez-Cruz G, Leon-Ortiz P et al (2016). Elevated myo-inositol, choline, and glutamate levels in the associative striatum of antipsychotic-naive patients with first-episode psychosis: a proton magnetic resonance spectroscopy study with implications for glial dysfunction. Schizophr Bull 42: 415-424.

Plitman E, Nakajima S, de la Fuente-Sandoval C, Gerretsen P, Chakravarty MM, Kobylianskii J et al (2014). Glutamatemediated excitotoxicity in schizophrenia: a review. Eur Neuropsychopharmacol 24: 1591-1605.

Provencher SW (2001). Automatic quantitation of localized in vivo $1 \mathrm{H}$ spectra with LCModel. NMR Biomed 14: 260-264.

Rimol LM, Hartberg CB, Nesvag R, Fennema-Notestine C, Hagler DJ Jr., Pung CJ et al (2010). Cortical thickness and subcortical volumes in schizophrenia and bipolar disorder. Biol Psychiatry 68: 41-50.

Rowland LM, Bustillo JR, Mullins PG, Jung RE, Lenroot R, Landgraf $\mathrm{E}$ et al (2005). Effects of ketamine on anterior cingulate glutamate metabolism in healthy humans: a 4 -T proton MRS study. Am J Psychiatry 162: 394-396.

Sled JG, Zijdenbos AP, Evans AC (1998). A nonparametric method for automatic correction of intensity nonuniformity in MRI data. IEEE Trans Med Imaging 17: 87-97.

Song X, Quan M, Lv L, Li X, Pang L, Kennedy D et al (2015). Decreased cortical thickness in drug naive first episode schizophrenia: in relation to serum levels of BDNF. J Psychiatr Res 60: 22-28.

Stone JM, Dietrich C, Edden R, Mehta MA, De Simoni S, Reed LJ et al (2012). Ketamine effects on brain GABA and glutamate levels with 1H-MRS: relationship to ketamine-induced psychopathology. Mol Psychiatry 17: 664-665.

Stone JM, Morrison PD, Pilowsky LS (2007). Glutamate and dopamine dysregulation in schizophrenia-a synthesis and selective review. J Psychopharmacol (Oxford, England) 21: 440-452.

Theberge J, Williamson KE, Aoyama N, Drost DJ, Manchanda R, Malla AK et al (2007). Longitudinal grey-matter and glutamatergic losses in first-episode schizophrenia. $\mathrm{Br} J$ Psychiatry 191: 325-334.

Tustison NJ, Avants BB, Cook PA, Zheng Y, Egan A, Yushkevich PA et al (2010). N4ITK: improved N3 bias correction. IEEE Trans Med Imaging 29: 1310-1320.

van Erp TG, Hibar DP, Rasmussen JM, Glahn DC, Pearlson GD, Andreassen OA et al (2016). Subcortical brain volume abnormalities in 2028 individuals with schizophrenia and 2540 healthy controls via the ENIGMA consortium. Mol Psychiatry 21: 585.

Venkatasubramanian G, Gangadhar BN, Jayakumar PN, Janakiramaiah N, Keshavan MS (2003). Reduced caudate volume in never-treated schizophrenia: evidence for neuro developmental etiopathogenesis. Indian J Psychiatry 45: 20-26.

Wijtenburg SA, Yang S, Fischer BA, Rowland LM (2015). In vivo assessment of neurotransmitters and modulators with magnetic resonance spectroscopy: application to schizophrenia. Neurosci Biobehav Rev 51: 276-295.

Xiao Y, Lui S, Deng W, Yao L, Zhang W, Li S et al (2015). Altered cortical thickness related to clinical severity but not the untreated disease duration in schizophrenia. Schizophr Bull 41: 201-210.

Zijdenbos AP, Forghani R, Evans AC (2002). Automatic "pipeline" analysis of 3-D MRI data for clinical trials: application to multiple sclerosis. IEEE Trans Med Imag 21: 1280-1291.

Supplementary Information accompanies the paper on the Neuropsychopharmacology website (http://www.nature.com/npp) 